\title{
Fundamental Relationship between Node Density and Delay in Wireless Ad Hoc Networks with Unreliable Links
}

\author{
Shizhen Zhao \\ Dept. of Elec. Engin. \\ Shanghai JiaoTong Univ. \\ Shanghai, China \\ zsz@sjtu.edu.cn \\ Xinbing Wang \\ Dept. of Elec. Engin. \\ Shanghai JiaoTong Univ. \\ Shanghai, China \\ xwang8@sjtu.edu.cn
}

\author{
Luoyi $\mathrm{Fu}$ \\ Dept. of Elec. Engin. \\ Shanghai JiaoTong Univ. \\ Shanghai, China \\ fly@sjtu.edu.cn \\ Qian Zhang \\ Dept. of Comp. Sci. and Engin \\ HK Univ. of Sci. and Tech. \\ Hong Kong, China \\ qianzh@cs.ust.hk
}

\begin{abstract}
We investigate the fundamental relationship between node density and transmission delay in large-scale wireless ad hoc networks with unreliable links from percolation perspective. Previous works[11][2][10] have already showed the relationship between transmission delay and distance from source to destination. However, it still remains as an open question how transmission delay varies in accordance with node density. Answering this question can provide guidance for determining the number of nodes to meet the delay requirement when designing ad hoc networks. In this paper, we study the impact of node density $\lambda$ on the ratio of delay and distance, denoted by $\gamma(\lambda)$. We analytically characterize the properties of $\gamma(\lambda)$ as a function of $\lambda$. And then we present upper and lower bounds to $\gamma(\lambda)$. Next, we take propagation delay into consideration and obtain further results on the upper and lower bounds of $\gamma(\lambda)$. Finally, we make simulations to verify our theoretical analysis.
\end{abstract}

\section{Categories and Subject Descriptors}

C.2.1 [Computer-Communication Networks]: Network Architecture and Design-Wireless Communications

\section{General Terms}

Theory and Performance

\section{Keywords}

Connectivity, Delay, Density

Permission to make digital or hard copies of all or part of this work for personal or classroom use is granted without fee provided that copies are not made or distributed for profit or commercial advantage and that copies bear this notice and the full citation on the first page. To copy otherwise, to republish, to post on servers or to redistribute to lists, requires prior specific permission and/or a fee.

MobiCom'11, September 19-23, 2011, Las Vegas, Nevada, USA.

Copyright 2011 ACM 978-1-4503-0492-4/11/09 ...\$5.00.

\section{INTRODUCTION}

Wireless communication sees an explosive growth in the number of customers in the past few decades, making largescale wireless ad hoc network an important part of modern life. In such a large-scale wireless network, information are transmitted in a multi-hop fashion. However, due to the unreliability of links in severe environment, such a multi-hop path may not exist all the time, making delay a significant issue. Besides, some power saving mechanism, e.x., turning off some base stations intermittently, can even exacerbate the delay performance. Therefore, it is important to study the relationship between node density and delay, so that we can control the delay in a desirable way.

To make our study meaningful, we assume the large-scale network is connnected. Full connectivity[3] can ensure the successful communication between node pairs in a wireless network, . However, it is overly power consuming to achieve full connectivity in large-scale networks $(i . e$. , the power required to maintain full connectivity increases with the size of the network). Thus, it is necessary to introduce a slightly weaker connectivity criterion, i.e., an infinite connected component containing a high fraction of the network nodes exists in a network. Thanks to percolation theory[9][14], it is possible to achieve this weaker connectivity in large-scale networks with power bounded.

Percolation theory[9], especially continuum percolation, has become a useful mathematical tool when analyzing the capacity and the connectivity of wireless networks. The most general model in Continuum Percolation, Random Connection Model(RCM), describes the behavior of connected clusters in a random geometric graph in which nodes are distributed according to poisson point process with node density $\lambda$, and two nodes share a link according to a connection function $h(r)$. A fundamental result of RCM points out a phase transition effect ${ }^{1}$. For $\lambda>\lambda_{c}$ (supercritical), there exist a unique connected component containing an infinite number of nodes(we also say the network is percolated). For $\lambda<\lambda_{c}$ (subcritical), all connected component in the network are finite almost surely.

\footnotetext{
${ }^{1}$ The condition for the existence of phase transition is $0<$ $\int_{\mathbb{R}^{2}} h(r) d r<\infty$.
} 
Applying percolation theory to wireless networks with unreliable links, we introduce two important concepts, i.e., instantaneous connectivity and long-term connectivity. Instantaneous connectivity requires wireless network percolated all the time. Long-term connectivity requires wireless network percolated in the long run(we will elaborate it more clearly later in section2.2.1). The instantaneous critical density, denoted by $\lambda_{I}$, is the critical density for instantaneous connectivity and the long-term critical density, denoted by $\lambda_{L}$, is the critical density for long-term connectivity. Long-term connectivity is a weaker criterion for connectivity, thus $\lambda_{L}<\lambda_{I}$. The Prerequisite for communication in wireless networks is connectivity, so we only focus on the case $\lambda>\lambda_{L}$.

In wireless ad hoc networks, delay is mainly composed of the waiting delay and the propagation delay. The waiting delay is caused by the lack of instantaneous connectivity. Information cannot be transmitted to a distant destination instantaneously since the connected component is finite almost surely. It must wait for some time until some communication links are established and can transmit forward again. Usually, such a waiting time is in the order of seconds, minutes or more. As for the propagation delay, it depends on the channel condition, scheduling and routing algorithm, arrival rate, etc. Mostly, the propagation delay is in the order of milliseconds if the network is not heavy loaded. Therefore, it is negligibly small compared to the waiting delay. For ease of analysis, we first ignore the impact of propagation delay and will consider its effect in the last.

Previous works [11][2][10] have showed that if $\lambda_{L}<\lambda<$ $\lambda_{I}$, the transmission delay scales linearly with distance between source and destination $(\gamma(\lambda)>0)$, and if $\lambda>\lambda_{I}$, the transmission delay scales sub-linearly with distance $(\gamma(\lambda)=$ $0)$. We can operate the network at $\lambda>\lambda_{I}$, but it is usually too costly and sometimes unnecessary to build so much nodes(base station). Another choice is to operate the network at $\lambda_{L}<\lambda<\lambda_{I}$. Then the cost deceases, however, if we want the network to meet some delay requirement for those important flooding information, simply knowing $\gamma(\lambda)>0$ is far from enough. Therefore, we need to know the exact value or the lower and upper bounds of $\gamma(\lambda)$ for ease of designing a proper network.

In this paper, we present a theoretical analysis about the delay in wireless networks with unreliable links. We present 3 properties of $\gamma(\lambda)$ as a function of $\lambda$. Using coupling techniques, we prove that $\gamma(\lambda)$ is a monotone decreasing function.

And then, we come to the upper and lower bounds of $\gamma(\lambda)$, while ignoring the propagation delay. For the upper bound, we first find a path between two nodes. And then we calculate the number of hops along this path and the delay at each hop. We obtain the result on upper bound through multiplication of the above two items. For the lower bound, we first introduce a concept called cluster to cluster transmission process and establish the relationship between delay and the cluster to cluster transmission process, which reveals the essence of delay in networks. Then, using the definition of delay of a cluster to cluster transmission, we obtain a lower bound of $\gamma(\lambda)$.

Next, we take propagation delay into consideration and reformulate $\gamma(\lambda)$ in this case. Propagation delay increases the delay in Large-Scale Networks, making $\gamma(\lambda)>0$ even when $\lambda>\lambda_{I}$. Using similar methods, we present new upper and lower bounds to $\gamma(\lambda)$ for all $\lambda>\lambda_{L}$.

Finally, we make enormous simulation and further verifies our theoretical results.

The original contributions that we have made in the paper are highlighted as follows:

- We present three properties to $\gamma(\lambda)$, i.e., $\gamma(\lambda)$ is uniformly bounded; $\gamma(\lambda)=0$ whenever $\lambda>\lambda_{I} ; \gamma(\lambda)$ is a monotone decreasing function.

- Ignoring propagation delay, we provide the upper bound and the lower bound to reflect the range of variation on $\gamma(\lambda)$.

- Taking propagation delay into consideration, we obtain further results.

- We conduct simulations to obtain experimental values of $\gamma(\lambda)$ in the above two cases. A new observation arises from our comparison between theoretical and simulation results is that the delay-distance ratio $\gamma(\lambda)$ can be estimated by the lower bound in relative dense networks while the experimental values of $\gamma(\lambda)$ get closer to the upper bound as $\lambda$ decreases. This also justifies the soundness of our theoretical conclusion.

The rest of the paper is organized as follows. In section 2 , we introduce our network model, several useful mathematical tools and some important notations. In section 3, we first give three properties of $\gamma(\lambda)$, and then present our main results concerning the upper and lower bound of $\gamma(\lambda)$. The analysis process to obtain the upper and lower bounds is given in section 4 . Simulation results are presented in Section 5 to support our theoretical findings. We summarize the paper in Section 6. Some proofs of the theorems and lemmas are presented in line or in Appendix.

\section{BACKGROUND AND NETWORK MODEL}

In this section, we present the background and network model. At first, we list some properties of poisson point process that are frequently used in this paper, and give a brief description of Random Connection Model. Then, we model the network as a random geometric graph, and define delay using first passage percolation model. Finally, we list some important notations in this paper.

\subsection{Background}

\subsubsection{Poisson Point Process}

In large-scale wireless networks with unreliable links, usually base stations cannot be placed anywhere due to severe environment. Or in the case that we have mobile stations, we cannot predict precisely the real time location of base stations. In these scenarios, a common way to model the location of base stations is to assume that those base stations are distributed uniformly in a given area, w.l.o.g., we assume $\lambda n^{2}$ base stations are evenly distributed in a $n \times n$ area. If we let $n \rightarrow \infty$, then the distribution of base stations will converge in distribution to Poisson Point Process 
with rate $\lambda^{2}$. The nice part of Poisson Point Process is the following two classical results on Poisson Point Process.

Lemma 2.1. [13]Let $\Gamma$ be a potentially inhomogeneous Poisson process on $\mathbb{R}^{d}$ with density function $\lambda(x)$, where $x=$ $\left(x_{1}, x_{2}, \ldots, x_{d}\right) \in \mathbb{R}^{d}$. Suppose that we obtain $\Gamma^{\prime}$ by independently coloring points $x \in \Gamma$ according to probabilities $p(x)$. Then $\Gamma^{\prime}$ and $\Gamma-\Gamma^{\prime}$ are two independent Poisson processes with density function $p(x) \lambda(x)$ and $(1-p(x)) \lambda(x)$, respectively.

Lemma 2.2. Let $\Gamma, \Gamma^{\prime}$ be two independent inhomogeneous Poisson process on $\mathbb{R}^{d}$ with density functions $\lambda(x)$ and $\lambda^{\prime}(x)$, respectively, where $x=\left(x_{1}, x_{2}, \ldots, x_{d}\right) \in \mathbb{R}^{d}$. Suppose that we obtain $\Gamma+\Gamma^{\prime}$ by superposing $\Gamma^{\prime}$ on $\Gamma$. Then $\Gamma+\Gamma^{\prime}$ is a Poisson processes with density function $\lambda(x)+\lambda^{\prime}(x)$.

The above two lemmas point out that the decomposition or superposition of Poisson Point Processes remains to be Poisson Point Process. This is very useful in coupling techniques. Moreover, we will use node and base station interchangeably if causing no confusion.

\subsubsection{Random Connection Model}

Before introducing the network model, we need a brief introduction to Continuum Percolation. Connectivity is a significant issue in wireless network, which has been extensively explored by [3][4][5][6][7][8] .In this part, we present the definition of connectivity in percolation perspective. To make the results in this paper applicable to more scenarios, we focus on the most general model in Continuum Percolation Theory, i.e., Random Connection Model(RCM).

In Random Connection Model, nodes are distributed according to Poisson point process $[12]$ in $\mathbb{R}^{d}$. Here we only focus on the case of $\mathbb{R}^{2}$ with node density $\lambda>0$. Each node $x$ connect to another node $y$ according to the connection function $h(r)$, where $r$ is the distance between $x$ and $y$, and $h(r)$ satisfy $0<\int_{\mathbb{R}^{2}} h(r) d r<\infty$.

We denote the RCM by $\mathcal{G}\left(\lambda, r_{0}, h(r)\right)$, where $\lambda$ is the node density, $r_{0}=\sup \{r \mid h(r)>0\}, \mathrm{h}(\mathrm{r})$ is the connection function. Then $\mathcal{G}\left(\lambda, r_{0}, h(r)\right)$ is a set of nodes connected by random links. For convenient, we assume the origin $0 \in \mathcal{G}\left(\lambda, r_{0}, h(r)\right)$.

Obviously, $\mathcal{G}\left(\lambda, r_{0}, h(r)\right)$ is composed of one or several disjointed connected clusters. Let us denote $W(A), A \subseteq$ $\mathcal{G}\left(\lambda, r_{0}, h(r)\right)$, the set of nodes attainable from nodes in set A, i.e.,

$$
W(A)=\left\{x \in \mathcal{G}\left(\lambda, r_{0}, h(r)\right) \mid \exists a \in A, a \leftrightarrow x\right\},
$$

where, $a \leftrightarrow x$ means that nodes $a$ and $x$ are in the same connected component.

Besides, we use $|W|$ to represent the cardinality of set $W$. And we write $\theta_{h}(\lambda)=\mathbb{P}_{\lambda, h}(|W(\{0\})|=\infty)^{3}$ and $\chi_{h}(\lambda)=$ $E_{\lambda, h}(|W(\{0\})|)^{4}$.

Then, the critical density can be determined in two ways, i.e.,

$$
\lambda_{\theta}(h)=\inf \left\{\lambda \mid \theta_{h}(\lambda)>0\right\} ;
$$

\footnotetext{
${ }^{2} \mathrm{We}$ fail to find the original statement in the related references. However, this statement can be justified by simply calculating the distribution of the number of base stations in a given area and then letting $n \rightarrow \infty$.

${ }^{3} \mathbb{P}$ is the probability of a event.

${ }^{4} E(x)$ is the expectation of random variable $x$
}

$$
\lambda_{\chi}(h)=\inf \left\{\lambda \mid \chi_{h}(\lambda)=\infty\right\} .
$$

According to Theorem 6.2 in [14], $\lambda_{\theta}(h)=\lambda_{\chi}(h)=\lambda_{c}(h)$. Furthermore, $0<\lambda_{c}(h)<\infty$. And there exists a unique infinite connected cluster if $\lambda>\lambda_{c}(h)$ (supercritical). This infinite connected cluster is also called the giant component, denoted by $\mathcal{C}\left(\mathcal{G}\left(\lambda, r_{0}, h(r)\right)\right)$. On the other hand, if $\lambda<$ $\lambda_{c}(h)$ (subcritical), all the connected components are finite almost surely.

Random Connection Model is just one kind of continuum percolation model. Another continuum percolation model is Poisson Boolean Model $\mathcal{B}(\lambda, r)$. In Poisson Boolean Model $\mathcal{B}(\lambda, r)$, nodes are distributed according to Poisson Point Process with density $\lambda$, and two nodes can communicate if and only if their distance is smaller than $r$. Poisson Boolean Model can also be seen as a collection of discs with radius $\frac{r}{2}$. Poisson Boolean Model is a special case of Random Connection Model, thus the conclusions for Random Connection Model still hold for Poisson Boolean Model.

\subsection{Model}

\subsubsection{Network Model}

We present the network model in this part. We model a large-scale network as a random geometric graph. Assume nodes are distributed according to Poisson Point Process with node density $\lambda$ in an infinite two-dimensional space $\mathbb{R}^{2}$. For each node $u$, we use $u$ to represent both this node and its location without causing confusion. We say two nodes share a link if and only if their distance is smaller than $r_{0}$. However, due to the severe natural hazards, enemy attack or energy depletion, each link suffers the possibility to fail. We model this failure as each link opening or closing intermittently.

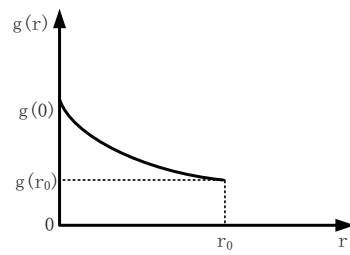

(a) Illustration of connection function $g(r)$.

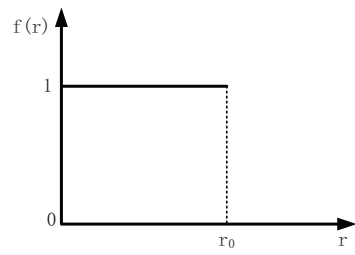

(b) Illustration of connection function $f(r)$.

\section{Figure 1: Illustration of two connection functions.}

Assume time is slotted. Consider a link with length $r$, at time slot $t$, we let it open with probability $g(r)$ (Fig. 1), independent of its former states. In reality, the farther two nodes are apart, the more difficult for a successful communication. Moveover, when $r>r_{0}$, there exists no link. Thus, it is reasonable to assume that $g(r)$ is a monotone decreasing function and $g(r)=0$ whenever $r>r_{0}$. Besides, we place another restriction on $g(r)$, i.e.,

$$
1>g(0) \geq g(r) \geq g\left(r_{0}\right)>0,0 \leq r \leq r_{0} .
$$

Then the network at each time slot $t$ can be represented by a Random Connection Model $\mathcal{G}_{t}\left(\lambda, r_{0}, g(r)\right)$. Here, we use subscript $t$ to indicate that the network is dynamic. Note that if $\lambda>\lambda_{c}(g(r)), \mathcal{G}_{t}\left(\lambda, r_{0}, g(r)\right)$ is percolated for all $t$ (we also say the network has instantaneous connectivity); while if $\lambda<\lambda_{c}(g(r)), \mathcal{G}_{t}\left(\lambda, r_{0}, g(r)\right)$ is not percolated for all $t$. Thus, the instantaneous critical density $\lambda_{I}=\lambda_{c}(g(r))$. 
Next, we introduce the concept of long-term connectivity. We first construct a new geometric graph. The location of all nodes in this graph is the same as that in $\mathcal{G}_{t}\left(\lambda, r_{0}, g(r)\right)$. Two nodes $x$ and $y$ share a link in this graph if and only if there exist $t$, such that $x$ and $y$ share an open link in $\mathcal{G}_{t}\left(\lambda, r_{0}, g(r)\right)$. Note that $x$ and $y$ has the potential to share a link in $\mathcal{G}_{t}\left(\lambda, r_{0}, g(r)\right)$ for some $t$, whenever $g(r)>0$ (equivalently, $r>r_{0}$ ). Thus this new geometric graph can be represented by a Random Connection Model $\mathcal{G}\left(\lambda, r_{0}, f(r)\right)$ (it can be also represented by Poisson Boolean Model $\left.\mathcal{B}\left(\lambda, r_{0}\right)\right)$. Here, $f(r)=1$ when $r<r_{0}$, and $f(r)=0$ when $r>r_{0}$ (Fig. 1). We say the wireless network has longterm connectivity if and only if $\mathcal{G}\left(\lambda, r_{0}, f(r)\right)$ is percolated. And the critical density $\lambda_{L}=\lambda_{c}(f(r))$ is defined as the long-term critical density.

As for the instantaneous critical density and the long-term critical density, we have the following relationship.

LEMMA 2.3. $\lambda_{L} \leq \lambda_{I}$. here.

The proof of this statement is quite trivial, so we omit it

Since the prerequisite for communication in large-scale wireless network is connectivity, it is enough to only focus on the case $\lambda>\lambda_{L}$.

\subsubsection{Modeling Delay in Large-Scale Networks}

This paper aims to find the relationship between node density and delay. The definition of delay of large-scale network is based on the First Passage Percolation. First Passage Percolation, first formulated by Hammersley and Welsh[1] in 1965, can be a very powerful tool for analysis of transmission delay in large-scale networks.

Given a Random Connection Model $\mathcal{G}\left(\lambda, r_{0}, h(r)\right)$, attach each link $e$ of $\mathcal{G}\left(\lambda, r_{0}, h(r)\right)$ a random variable $T_{c}(e)$, representing the time needed to pass through the link $e$. Consider a path $\pi$, the passage time is defined as

$$
T_{p}(\pi)=\sum_{e \in \pi}\left(T_{c}(e)\right) .
$$

And for any two nodes $x$ and $y(x, y$ are not necessarily adjacent), the first-passage time $T_{\lambda}(x, y)$ is given by

$$
T_{\lambda}(x, y)=\inf \left\{T_{p}(\pi): \pi \text { is a pass from } x \text { to } y\right\} .
$$

In this paper, the time needed to cross a link is composed of two parts. The dominating part is called waiting delay, which is caused by the unreliability of links. Sometimes, information must wait at one end of a link until this link is on. The unreliability of a link may be caused by a sudden wind, a sudden rain, etc. Usually, a link is more likely to remain in a state(good or bad) for several seconds, minutes, or even longer. The time slot in this paper is an enough long period for links to change states.

Another part of delay is caused by the bandwidth, scheduling and routing algorithm, the actual propagation speed. Usually such kind of delay is in the order of milliseconds, which is negligible small compared to the waiting delay. We don't want to go deep into this part. At first, we just ignore this part of delay. After that, when we are studying the impact of this part of delay, we simply model it as the propagation delay. We assume all active links can transmit simultaneously. Because the interference constraints is just equivalent to increasing the propagation delay a little bit.
We first only focus on the waiting delay. This is equivalent to introducing a random crossing time $T_{c}(e)$ to each link $e \in \mathcal{G}\left(\lambda, r_{0}, f(r)\right)$. Assume that the length of the link $e$ is $0<r<r_{0}, T_{c}(e)$ satisfy the Geometric distribution(here we have ignored the propagation delay),i.e.,

$$
\mathbb{P}\left(T_{c}(e)=k\right)=(1-g(r))^{k} g(r) .
$$

Eqn. (3) assures that $0<E\left(T_{c}(e)\right)<\infty$. Using Liggett's subadditive ergodic theorem[15], previous works have proved that, for $x, y \in \mathcal{C}\left(\mathcal{G}\left(\lambda, r_{0}, f(r)\right)\right)$, when $\lambda>\lambda_{L}$,

$$
\lim _{d(x, y) \rightarrow \infty} \frac{T_{\lambda}(x, y)}{d(x, y)}=\gamma(\lambda) .
$$

Moveover, if $\lambda_{L}<\lambda<\lambda_{I}, \gamma>0$; while if $\lambda>\lambda_{I}, \gamma=0$.

Eqn. (5) is also the definition of $\gamma(\lambda)$. From the former result, we can also see that $\gamma$ must depend on $\lambda$. However, the existing results only point out when $\gamma(\lambda)$ equals to 0 , and when it is larger than 0 . The exact relationship between $\gamma(\lambda)$ and $\lambda$ still remains as an open question. In this paper, we will give a more precise description on $\gamma(\lambda)$.

\subsection{Useful Notations}

Some useful notations are listed as follows.

- (Section 2.1.2) $\mathcal{G}\left(\lambda, r_{0}, h\right)$ is a Random Connection Model, and $h$ is the connection function; $\mathcal{B}(\lambda, r)$ is the Poisson Boolean Model; we use $\mathcal{C}\left(\mathcal{G}\left(\lambda, r_{0}, h\right)\right)$ and $\mathcal{C}(\mathcal{B}(\lambda, r))$ to represent the giant component of $\mathcal{G}\left(\lambda, r_{0}, h\right)$ and $\mathcal{B}(\lambda, r)$ respectively.

- (Section 2.2.1) $\mathcal{G}_{t}\left(\lambda, r_{0}, g\right)$ is the instantaneous geometric graph at time slot $t$ and its critical density is $\lambda_{I}$; $\mathcal{G}\left(\lambda, r_{0}, f\right)$ is the long-term geometric graph and its critical density is $\lambda_{L}$.

- $\mathbb{P}(\bullet)$ represents the probability of some event; $E(\bullet)$ represents the expectation of a random variable; $z_{x}\left(z_{y}\right)$ represents the $x(y)$-coordinate of $z ; d(u, v)=\|u-v\|$ is the Euclidean distance between node $u$ and $v$.

- (Section 4.3)H( $\left.z_{0}, a\right)$ is a circular region defined as $H\left(z_{0}, a\right)=\left\{z=\left(z_{x}, z_{y}\right) \in \mathbb{R}^{2} \mid\left\|z-z_{0}\right\|<a\right\}$. The random variable $S_{g, t, u}(\lambda)$ is defined as $S_{g, t, u}(\lambda)=$ $\sup \left\{a \mid \exists\right.$ node $v \in H^{c}(u, a), v$ and $u$ are connected at time slot $t\}$. Actually, the distribution of $S_{g, t, u}(\lambda)$ is independent of $t$ and $u$. Thus, we write $S_{g, t, u}(\lambda)$ as $S_{g}(\lambda)$ for short.

- (Section 2.2.2) $T_{c}(e)$ is the passage time for a link $e$; $T_{p}(\pi)$ is the passage time for a path $\pi ; T_{\lambda}(x, y)$ is the first passage time from node $x$ to $y$; (Section 4.2) $T_{p}(\Pi)$ is the passage time for a cluster to cluster transmission process $\Pi$;

- (Section 4.1) $N_{\lambda}(d(u, v))$ is the minimum number of hops from node $u$ to $v$.

- $\pi$ represents a path; $\Pi$ represents a cluster to cluster transmission process. 


\section{MAIN RESULTS}

In this section, we first give some properties on the delaydistance ratio $\gamma(\lambda)$. And then we present our main results concerning the tradeoff between node density and $\gamma(\lambda)$ in wireless networks with unreliable links, in which an upper bound and a lower bound for $\gamma(\lambda)$, are given.

\subsection{Properties of $\gamma(\lambda)$}

$\gamma(\lambda)$ can be seen as a function mapping from $\left[\lambda_{L}, \infty\right)$ to

$\mathbb{R}$. The properties of $\gamma(\lambda)$ are listed below.

THEOREM 3.1. $\gamma(\lambda)$ has the following three properties:

- there exists $\gamma_{M}<\infty$, such that for $\forall \lambda, \gamma(\lambda) \leq \gamma_{M}$;

- $f \circ r \forall \lambda>\lambda_{I}, \gamma(\lambda)=0$;

- $\gamma(\lambda)$ is a monotone decreasing function.

PROOF. The first property can be proved later, so we do not elaborate it here. The second property has already been proved by previous literatures[11] [10][2][16]. Thus, we only present the proof of property 3 here.

Given $\lambda_{1}>\lambda_{2}$, consider two Random Connection Models $\mathcal{G}_{t}\left(\lambda_{1}, r_{0}, g(r)\right)$ and $\mathcal{G}_{t}\left(\lambda_{2}, r_{0}, g(r)\right)$. We use coupling technique to prove $\gamma\left(\lambda_{1}\right) \leq \gamma\left(\lambda_{2}\right)$.

Note that nodes in $\mathcal{G}_{t}\left(\lambda_{1}, r_{0}, g(r)\right)$ and $\mathcal{G}_{t}\left(\lambda_{2}, r_{0}, g(r)\right)$ are distributed according to Poisson Point Process $\Gamma_{1}$ and $\Gamma_{2}$ with node densities $\lambda_{1}$ and $\lambda_{2}$, respectively. According to lemma 2.2, $\Gamma_{1}$ can be seen as the superposition of $\Gamma_{2}$ and another Poisson Point Process $\Gamma^{\prime}$ with node density $\lambda_{2}-\lambda_{1}$.

Consider nodes $x, y \in \Gamma_{2}$, since $\Gamma_{2} \subseteq \Gamma_{1}$, we obtain $x, y \in$ $\Gamma_{1}$. For any path $\pi$ connecting $x$ and $y$ in $\mathcal{G}_{t}\left(\lambda_{2}, r_{0}, g(r)\right)$, this path also exists in $\mathcal{G}_{t}\left(\lambda_{1}, r_{0}, g(r)\right)$. And the delay from $x$ to $y, T_{\lambda}(x, y)$, is defined as the minimum delay among all paths connecting $x$ and $y$. Thus,

$$
T_{\lambda_{1}}(x, y) \leq T_{\lambda_{2}}(x, y)
$$

Divide the above inequality by $d(x, y)$, and let $d(x, y) \rightarrow$ $\infty$, we obtain

$$
\gamma\left(\lambda_{1}\right) \leq \gamma\left(\lambda_{2}\right)
$$

We also have a conjecture about $\gamma(\lambda)$, i.e., $\gamma(\lambda)$ is a continuous function. However, we fail to prove it.

According to Theorem 3.1, we can sketch $\gamma(\lambda)$ out(Fig. 2).

\subsection{Main results on $\gamma(\lambda)$}

We have obtained several properties of $\gamma(\lambda)$. Now we are ready to present our main results.

Theorem 3.2. Given a $R C M \mathcal{G}_{t}\left(\lambda, r_{0}, g(r)\right)$ with $\lambda_{L}<$ $\lambda<\lambda_{I}$, the corresponding $\gamma(\lambda)$ satisfies

$$
\frac{1}{E\left(S_{g}(\lambda)+r_{0}\right)} \leq \gamma(\lambda) \leq \inf _{\lambda^{\prime} \in\left[\lambda_{L}, \lambda\right]} \kappa \sqrt{\frac{\lambda^{\prime}}{\lambda_{L}}}\left(\frac{1}{g\left(r_{0} \sqrt{\frac{\lambda_{L}}{\lambda^{\prime}}}\right)}-1\right),
$$

where $\kappa$ is a constant independent of $\lambda$.

If we take propagation delay into consideration, we have the following results.

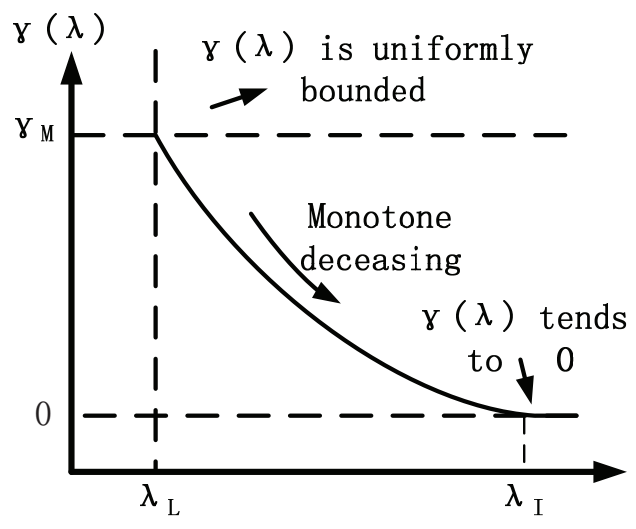

Figure 2: Sketch of $\gamma(\lambda)$ (without considering propagation delay).

THEOREM 3.3. Given a $R C M \mathcal{G}_{t}\left(\lambda, r_{0}, g(r)\right)$ with $\lambda>\lambda_{L}$, $\tau$ is the propagation delay for a existing link and $\tau<1$. Then the corresponding $\gamma(\lambda)$ satisfies

$$
\frac{1}{E\left(\min \left\{S_{g}(\lambda), \frac{r_{0}}{\tau}\right\}\right)+r_{0}} \leq \gamma(\lambda) \leq \inf _{\lambda^{\prime} \in\left[\lambda_{L}, \lambda\right]} \frac{\kappa \sqrt{\lambda^{\prime} / \lambda_{L}}}{g\left(r_{0} \sqrt{\frac{\lambda_{L}}{\lambda^{\prime}}}\right)}
$$

where $\kappa$ is a constant independent of $\lambda$.

Our results provide a theoretical way to estimate delay in large-scale wireless networks. We use connection function $g(r)$ to represent the condition of a large-scale wireless network, making our results applicable to most cases in real networks. Also, waiting delay and propagation delay are both taken into account in our formulation, making our results more reliable.

\section{UPPER AND LOWER BOUNDS OF $\gamma(\lambda)$}

In this section, we first give an upper bound to the delaydistance ratio, $\gamma(\lambda)$. And then, we make further analysis on transmission delay and introduce a concept called cluster to cluster transmission. Using this concept, we derive a lower bound. Finally, we take propagation delay into consideration, and formulate its impact on $\gamma(\lambda)$.

Turn back to the definition of $\gamma(\lambda)($ Eqn. 5).

$$
\gamma(\lambda)=\lim _{d(x, y) \rightarrow \infty} \frac{T_{\lambda}(x, y)}{d(x, y)}
$$

where $x, y$ belong to the giant component of $\mathcal{G}\left(\lambda, r_{0}, f(r)\right)$. However, we needn't calculate the limit for all pairs of $x, y \in$ $\mathcal{C}\left(\mathcal{G}\left(\lambda, r_{0}, f(r)\right)\right)$. The correctness of this assertion is assured by the following lemma.

LEMMA 4.1. Given a convergent sequence $\left\{x_{k}\right\}, k=1,2, \ldots$, and $\lim _{k \rightarrow \infty} x_{k}=x_{0} .\left\{y_{k}\right\}, k=1,2, \ldots$, is a subsequence of $\left\{x_{k}\right\}$, and $\lim _{k \rightarrow \infty} y_{k}=y_{0}$. Then $x_{0}=y_{0}$.

Obviously, the number of nodes in $\mathcal{C}\left(\mathcal{G}\left(\lambda, r_{0}, f(r)\right)\right)$ is countable. We enumerate for all nodes. We randomly select a node and label it as $x_{0}$, and then label other nodes according to the distance from $x_{0}$ (larger subscript means larger distance from $\left.x_{0}\right)$. Define sequence $\left\{m_{k}, k=1,2, \ldots,\right\}, m_{k}=$ 
$\frac{T_{\lambda}\left(x_{0}, x_{k}\right)}{d\left(x_{0}, x_{k}\right)}$, then $\lim _{k \rightarrow \infty} m_{k}=\gamma(\lambda)$. According to lemma 4.1 , we only need to find a subset of nodes of $\mathcal{C}\left(\mathcal{G}\left(\lambda, r_{0}, f(r)\right)\right)$ (the cardinality of this subset must be infinity), and calculate $\gamma(\lambda)$ from this subset. This technique is used in deriving the upper bound.

\subsection{Upper Bound of $\gamma(\lambda)$}

The definition of of delay is defined as the minimum delay along all paths connecting source and destination nodes. Thus, it must be smaller than or equal to the delay along one path. In this part, we will first find a subset of nodes of $\mathcal{C}\left(\mathcal{G}\left(\lambda, r_{0}, f(r)\right)\right)$. And then we find a path for each node pair in this subset. After that, we calculate the delay along this path. Finally, dividing the delay by distance, we obtain an upper bound of $\gamma(\lambda)$.

Before proceeding, we need the following lemma.

Lemma 4.2. Consider Poisson Boolean models in $\mathbb{R}^{2}$. Let $\lambda_{c}(r)$ denote the critical density in the case where the transmission range is $r$. Then it is the case that

$$
\lambda_{c}\left(r_{1}\right) r_{1}^{2}=\lambda_{c}\left(r_{2}\right) r_{2}^{2},
$$

where $r_{1}, r_{2}>0$.

Proof. See Proposition 2.10 in [14].

The long-term critical density $\lambda_{L}$ is also the critical density of Poisson Boolean Model with transmission range $r_{0}$. Consider the network with density $\lambda>\lambda_{L}$, according to lemma 4.2, we immediately know that when $\lambda r^{2}>\lambda_{L} r_{0}^{2}$, i.e., $r>\sqrt{\frac{\lambda_{L}}{\lambda}} \cdot r_{0}$, Poisson Boolean Model $\mathscr{B}(\lambda, r)$ is percolated.

Let $0<\varepsilon<\sqrt{\frac{\lambda}{\lambda_{L}}}-1$ and $\tilde{r}=r_{0} \sqrt{\frac{\lambda_{L}}{\lambda}}(1+\epsilon)$, then $\mathcal{B}(\lambda, \tilde{r})$ is percolated. Note that, in Random Connection Model $\mathcal{G}\left(\lambda, r_{0}, f(r)\right)$, the transmission range is $r_{0}>\tilde{r}$. Thus, $\mathcal{B}(\lambda, \tilde{r})$ is a subgraph of $\mathcal{G}\left(\lambda, r_{0}, f(r)\right)$. We denote the giant component of $\mathcal{B}(\lambda, \tilde{r})$ by $\mathcal{C}(\mathcal{B}(\lambda, \tilde{r}))$. According to the uniqueness of giant component in supercritical case, there must be $\mathcal{C}(\mathcal{B}(\lambda, \tilde{r})) \subseteq \mathcal{C}\left(\mathcal{G}\left(\lambda, r_{0}, f(r)\right)\right)$. According to lemma 4.1, when calculating $\gamma(\lambda)$, we only need to focus on the case that both nodes belong to $\mathcal{C}(\mathcal{B}(\lambda, \tilde{r}))$.

Assume that nodes $u, v \in \mathcal{C}(\mathcal{B}(\lambda, \tilde{r}))$. Then there exists at least one path in $\mathcal{B}(\lambda, \tilde{r})$ from $u$ to $v$. We choose the path with minimum number of hops, and denote it by $\pi_{m}$.

Up to now, we have found a path connecting $u$ and $v$. Next, we are to calculate the delay along this path. To start with, we need to work out the number of hops, denoted by $N_{\lambda}(d(u, v))$, in $\pi_{m}$. We needn't calculate each $N_{\lambda}(d(u, v))$ for different $\lambda$. We can find the relationship of $N_{\lambda}(d(u, v))$ for different $\lambda$ in the following way.

Scale the network up by $\sqrt{\frac{\lambda}{\lambda_{L}}}$, and then the node density becomes $\lambda_{L}$, the transmission range becomes $(1+\epsilon) r_{0}$, and the distance between $u$ and $v$ becomes $d(u, v) \sqrt{\frac{\lambda}{\lambda_{L}}}$. As a result, calculating $N_{\lambda}(d(u, v))$ is equivalent to calculating $N_{\lambda_{L}}\left(d(u, v) \sqrt{\frac{\lambda}{\lambda_{L}}}\right)$. Next, we present the lemma concerning $N_{\lambda_{L}}(d)$.

Lemma 4.3. Given $\mathcal{B}\left(\lambda_{L},(1+\epsilon) r_{0}\right)$, and $u, v \in \mathcal{C}\left(\mathcal{B}\left(\lambda_{L}\right.\right.$ $, 1+\epsilon)$ ), the minimal number of hops needed for transmitting information from $u$ to $v$ is $N_{\lambda_{L}}(d(u, v))$. Then there exist $\kappa$ such that

$$
\lim _{d(u, v) \rightarrow \infty} \frac{N_{\lambda_{L}}(d(u, v))}{d(u, v)}=\kappa .
$$

The proof of lemma 4.3 uses Liggett's subadditive ergodic theorem. This technique has already been explored in [2][10]. For detailed proof, please refer to our technical report [17].

According to lemma 4.3, we immediately get

$$
\begin{aligned}
\lim _{d(u, v) \rightarrow \infty} \frac{N_{\lambda}(d(u, v))}{d(u, v)} & =\lim _{d(u, v) \rightarrow \infty} \frac{N_{\lambda_{L}}\left(d(u, v) \sqrt{\frac{\lambda}{\lambda_{L}}}\right)}{d(u, v)} \\
& =\kappa \sqrt{\frac{\lambda}{\lambda_{L}}}
\end{aligned}
$$

Then we calculate the delay $T_{p}\left(\pi_{m}\right)$ along path $\pi_{m}$. According to Strong Large Number Theory, with high probability, we have

$$
T_{p}\left(\pi_{m}\right)=\sum_{e \in \pi_{m}} T_{c}(e)=N_{\lambda}(d) E\left[T_{c}(e)\right] .
$$

Therefore,

$$
\begin{aligned}
\gamma(\lambda) & =\lim _{d \rightarrow \infty} \frac{T_{\lambda}(u, v)}{d(u, v)} \\
& \leq \lim _{d \rightarrow \infty} \frac{T_{p}\left(\pi_{m}\right)}{d(u, v)} \\
& =E\left[T_{c}(e)\right] \lim _{d \rightarrow \infty} \frac{N_{\lambda}(d(u, v))}{d(u, v)} \\
& =\kappa \sqrt{\frac{\lambda}{\lambda_{L}}} E\left[T_{c}(e)\right] .
\end{aligned}
$$

From the definition of the path $\pi_{m}$, we know that the length of each hop is smaller than $\tilde{r}=r_{0} \sqrt{\frac{\lambda_{L}}{\lambda}}(1+\epsilon)$. Besides, the connection function $g(r)$ is monotone decreasing. Thus, for a link $e^{\prime}$ whose length is $\tilde{r}$, there must be

$$
\begin{aligned}
E\left[T_{c}(e)\right] & \leq E\left[T_{c}\left(e^{\prime}\right)\right] \\
& =\sum_{k=0}^{\infty} k \mathbb{P}\left(T_{c}\left(e^{\prime}\right)=k\right) \\
& =\sum_{k=0}^{\infty} k(1-g(\tilde{r}))^{k} g(\tilde{r}) \\
& =\frac{1}{g(\tilde{r})}-1 .
\end{aligned}
$$

Thus,

$$
\begin{aligned}
\gamma(\lambda) & =\kappa \sqrt{\frac{\lambda}{\lambda_{L}}} E\left[T_{c}(e)\right] \\
& \leq \kappa \sqrt{\frac{\lambda}{\lambda_{L}}}\left(\frac{1}{g(\tilde{r})}-1\right) \\
& =\kappa \sqrt{\frac{\lambda}{\lambda_{L}}}\left(\frac{1}{g\left(r_{0} \sqrt{\frac{\lambda_{L}}{\lambda}}(1+\epsilon)\right)}-1\right) .
\end{aligned}
$$

Let $\epsilon \rightarrow 0$, we obtain

$$
\gamma(\lambda) \leq \kappa \sqrt{\frac{\lambda}{\lambda_{L}}}\left(\frac{1}{g\left(r_{0} \sqrt{\frac{\lambda_{L}}{\lambda}}\right)}-1\right) .
$$


Furthermore, from property 3 of theorem 3.1, we know $\gamma(\lambda)$ is a monotone decreasing function. Thus,

$$
\gamma(\lambda) \leq \inf _{\lambda^{\prime} \in\left[\lambda_{L}, \lambda\right]} \kappa \sqrt{\frac{\lambda^{\prime}}{\lambda_{L}}}\left(\frac{1}{g\left(r_{0} \sqrt{\frac{\lambda_{L}}{\lambda^{\prime}}}\right)}-1\right) .
$$

\subsection{Cluster to Cluster Transmission}

In section 4.1, we have obtained the upper bound of $\gamma(\lambda)$ by calculating the delay along one path. However, the method used in section 4.1 cannot be generalized to study the lower bound of $\gamma(\lambda)$. We don't know the number of paths connecting two nodes, nor do we know the delay along each path. Thus, it is impossible to find the minimum delay along all the paths.

To find a lower bound of $\gamma(\lambda)$, we need to make clear that when delay is introduced to network.

Consider transmitting information from node $u$ to $v$. Assume that at time slot $t_{1}$, node $u_{1}\left(u_{1}=u\right)$ transmits information to other nodes. Since we have ignored the propagation delay, all nodes connected to $u_{1}$ in geometric graph $\mathcal{G}_{t_{1}}\left(\lambda, r_{0}, g(r)\right)$, denoted by $w_{1}$, receive the information instantaneously. Then, the transmission process stops. The transmission process will restart at time slot $t_{2}>t_{1}{ }^{5}$, when at least one node in $w_{1}$ find the opportunity to forward the information to a new node, denoted by $u_{2}$. At this time slot, $u_{2}$ transmits information to those nodes which are connected to $u_{2}$ in geometric graph $\mathcal{G}_{t_{2}}\left(\lambda, r_{0}, g(r)\right)$, and do not belong to $w_{1}$, denoted by $w_{2}$, instantaneously. This process goes on, until at time slot $t_{M}$, node $u_{M}$ and the destination node $v$ are in the same connected cluster and information is transmitted to node $v$ instantaneously.

We can see that the cluster to cluster transmission as a series of outbursts. During each outburst, some new nodes receive the information. $w_{k}, k=1,2, \ldots, M$ is the set of nodes which receive the information right at the $k$ th outburst. A cluster to cluster transmission process can be represented by $\Pi=\left\{\left(t_{1}, u_{1}\right),\left(t_{2}, u_{2}\right), \ldots,\left(t_{M}, u_{M}\right)\right\}$. Information can be transmitted from $u$ to $v$ through $\Pi$. Define the passage time for the cluster to cluster transmission process $\Pi$ as

$$
T_{p}(\Pi)=t_{M}-t_{1} .
$$

Then, we have the following lemma.

Lemma 4.4. Given nodes $u, v \in \mathcal{C}\left((G)\left(\lambda, r_{0}, f(r)\right)\right)$, the first passage time

$$
\begin{array}{r}
T_{\lambda}(u, v)=\inf \left\{T_{p}(\Pi) \mid \Pi\right. \text { is a cluster to cluster } \\
\text { transmission process from } u \text { to } v\} .
\end{array}
$$

Proof. For convenience, we use $\mathscr{L}$ to denote the set of cluster to cluster transmission process from $u$ to $v$. Then Eqn.(11) can be rewritten as

$$
T_{\lambda}(u, v)=\inf \left\{T_{p}(\Pi) \mid \Pi \in \mathscr{L}\right\} .
$$

It is easy to see that for each cluster to cluster transmission process $\Pi$ from $u$ to $v$,

$$
T_{p}(\Pi) \geq T_{\lambda}(u, v)
$$

\footnotetext{
${ }^{5}$ Here, we do not require $t_{2}$ to be the smallest, i.e., there may exist $t_{1}<t^{\prime}<t_{2}$, such that at least one node in $w_{1}$ have the opportunity to forward the information to a new node at time slot $t^{\prime}$.
}

Thus,

$$
\inf \left\{T_{p}(\Pi) \mid \Pi \in \mathscr{L}\right\} \geq T_{\lambda}(u, v) .
$$

Next, we show that

$$
\inf \left\{T_{p}(\Pi) \mid \Pi \in \mathscr{L}\right\} \leq T_{\lambda}(u, v) .
$$

Recall the definition of $T_{\lambda}(u, v)$, i.e.,

$$
T_{\lambda}(x, y)=\inf \left\{T_{p}(\pi): \pi \text { is a pass from } x \text { to } y\right\} .
$$

Let $\pi_{0}$ be the path with minimum delay from $u$ to $v$, we prove that there exists a cluster to cluster transmission process $\Pi_{0}$, such that $T_{p}\left(\Pi_{0}\right)=T_{p}\left(\pi_{0}\right)$.

Assume that $\pi_{0}=i_{0} i_{1} i_{2} \ldots i_{K}\left(i_{0}=u, i_{K}=v\right)$. At time slot $t_{1}$, some nodes in path $\pi_{0}$ may be in the same connected cluster as $i_{0}$. Let $i_{\eta_{1}-1}$ be the node attainable from $i_{0}$ with largest subindex, then the link between $i_{\eta_{1}-1}$ and $i_{\eta_{1}}$ must be off at this time slot. Let $t_{2}>t_{1}$ be the first time slot that this link is on. At time slot $t_{2}$, let $i_{\eta_{2}-1}$ be the node attainable from $i_{\eta_{1}}$ with largest subindex. Then the link between $i_{\eta_{2}-1}$ and $i_{\eta_{2}}$ must be off until time slot $t_{3}>t_{2} \ldots$ At time slot $t_{k}$, node $i_{\eta_{k-1}}$ and destination node $v$ are in the same connected cluster and the information transmit to $v$ instantaneously. We denote by $\Pi_{0}$ this cluster to cluster transmission process. And

$$
\Pi_{0}=\left\{\left(t_{1}, i_{0}\right),\left(t_{2}, i_{\eta_{1}}\right), \ldots,\left(t_{k}, i_{\eta_{k-1}}\right)\right\} .
$$

From the construction of $\Pi_{0}$, it is obvious that $T_{p}\left(\Pi_{0}\right)=$ $T_{p}\left(\pi_{0}\right)$.

Thus,

$$
\begin{aligned}
T_{\lambda}(u, v) & =T_{p}\left(\pi_{0}\right) \\
& =T_{p}\left(\Pi_{0}\right) \\
& \geq \inf \left\{T_{p}(\Pi) \mid \Pi \in \mathscr{L}\right\} .
\end{aligned}
$$

Combining Eqn.(12) and Eqn.(13), we obtain

$$
T_{\lambda}(u, v)=\inf \left\{T_{p}(\Pi) \mid \Pi \in \mathscr{L}\right\} .
$$

Lemma 4.4 establishes the relationship between delay and cluster to cluster transmission process. Cluster to cluster transmission process can represent the information dissemination process more precisely. Our following results on the lower bound of $\gamma(\lambda)$ are just based on the cluster to cluster transmission.

\subsection{Lower Bounds of $\gamma(\lambda)$}

In this section, we use the concept of cluster to cluster transmission process to derive a lower bound of $\gamma(\lambda)$.

To start with, we need to introduce a random variable $S_{g, t, u}(\lambda)(g$ is the connection function, $u$ is a node) to represent the size of connected cluster in the instantaneous geometric random graph $\mathcal{G}_{t}\left(\lambda, r_{0}, g(r)\right)$. We establish a cartesian coordinate in $\mathbb{R}^{2}$. We define $H\left(z_{0}, a\right)$ as

$$
H\left(z_{0}, a\right)=\left\{z=\left(z_{x}, z_{y}\right) \in \mathbb{R}^{2} \mid\left\|z-z_{0}\right\|<a\right\} .
$$

The random variable $S_{g, t, u}(\lambda)$ is defined as

$S_{g, t, u}(\lambda)=\sup \{a \mid \exists$ node $v \in H(u, a), v \leftrightarrow u$ at time slot $t\}$.

According to the translation invariance and time independence of our dynamic random connection model $\mathcal{G}_{t}\left(\lambda, r_{0}, g(r)\right)$, $S_{g, t, u}(\lambda)$ is independent of $t$ and $u$. Thus, we can write $S_{g, t, u}(\lambda)$ as $S_{g}(\lambda)$ if causing no confusion. 


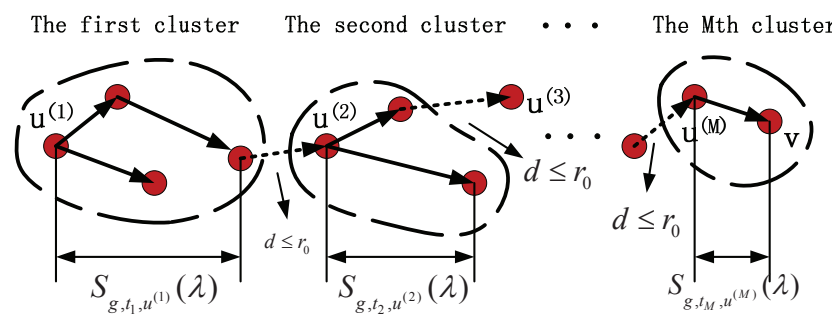

Figure 3: Illustration of a cluster to cluster transmission process.

Now, given two nodes $u$ (source) and $v$ (destination), consider a cluster to cluster transmission process(Fig. 3)

$$
\Pi=\left\{\left(u^{(1)}, t_{1}\right),\left(u^{(2)}, t_{2}\right), \ldots,\left(u^{(M)}, t_{M}\right)\right\},
$$

where $u^{(1)}=u$, and $u^{(M)}, v$ are in the same connected cluster at time slot $t_{M}$. Then the delay along this cluster to cluster transmission process is

$$
T_{p}(\Pi)=t_{M}-t_{1}=\sum_{k=1}^{M-1}\left(t_{k+1}-t_{k}\right) \geq M-1 .
$$

Note that, for $\forall k=1,2, \ldots, M-1, u^{(k+1)}$ is connected to a node in $w_{k}$, denoted by $u^{\prime}$. Then

$$
\begin{aligned}
\left\|u^{(k+1)}-u^{(k)}\right\| & \leq\left\|u^{(k+1)}-u^{\prime}\right\|+\left\|u^{\prime}-u^{(k)}\right\| \\
& \leq S_{g, t_{k}, u^{(k)}}(\lambda)+r_{0} .
\end{aligned}
$$

And for $k=M$,

$$
\left\|v_{x}-u^{(M)}\right\| \leq S_{g, t_{M}, u}(M)(\lambda) .
$$

Combining the above two inequalities together, we obtain,

$$
\begin{aligned}
d(u, v) & =\|v-u\| \\
& \leq \sum_{k=1}^{M-1}\left\|u^{(k+1)}-u^{(k)}\right\|+\left\|v-u^{(M)}\right\| \\
& \leq \sum_{k=1}^{M-1}\left(S_{g, t_{k}, u^{(k)}}(\lambda)+r_{0}\right)+S_{g, t_{M}, u_{x}^{(M)}}(\lambda) \\
& <\sum_{k=1}^{M}\left(S_{g, t_{k}, u^{(k)}}(\lambda)+r_{0}\right) .
\end{aligned}
$$

For $\forall k, S_{g, t_{k}, u^{(k)}}(\lambda)$ admit the same distribution as $S_{g}(\lambda)$. Moreover, according to the spatial independence of Poisson Point Process, $S_{g, t_{k}, u}(k)(\lambda)$ are i.i.d. random variables. According to the law of strong large numbers, we have

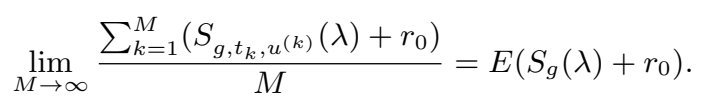

That is, for $\forall \epsilon>0, \exists M_{\epsilon}$, such that $\forall M>M_{\epsilon}$ (this condition is satisfied for large enough $d(u, v))$, we have

$$
\frac{\sum_{k=1}^{M}\left(S_{g, t_{k}, u^{(k)}}(\lambda)+r_{0}\right)}{M}<E\left(S_{g}(\lambda)+r_{0}\right)+\epsilon .
$$

Combined with Eqn. (14), we have

$$
d(u, v)<M\left(E\left(S_{g}(\lambda)+r_{0}\right)+\epsilon\right) .
$$

Then

$$
T_{p}(\Pi) \geq M-1>\frac{d(u, v)}{E\left(S_{g}(\lambda)+r_{0}\right)+\epsilon}-1 .
$$

Note that the right part of the above equation does not depend on the selection of the cluster to cluster transmission. Thus,

$$
T_{\lambda}(u, v) \geq \frac{d(u, v)}{E\left(S_{g}(\lambda)+r_{0}\right)+\epsilon}-1 .
$$

Therefore,

$$
\begin{aligned}
\gamma(\lambda) & =\lim _{d(u, v) \rightarrow \infty} \frac{T_{\lambda}(u, v)}{d(u, v)} \\
& \geq \frac{1}{E\left(S_{g}(\lambda)+r_{0}\right)+\epsilon}
\end{aligned}
$$

Let $\epsilon \rightarrow 0$, we finally obtain

$$
\gamma(\lambda) \geq \frac{1}{E\left(S_{g}(\lambda)+r_{0}\right)}
$$

\subsection{Impact of Propagation Delay}

The delay in a large-scale wireless network is composed of two parts, i.e., the waiting delay and the propagation delay. In previous sections, we have formulated the waiting delay, while ignoring the propagation delay. However, propagation delay may become dominant in some cases especially when the node density is large enough ${ }^{6}$. In the following discussion, we denote by $\tau$ the propagation delay for a existing link. For ease of analysis, we assume that the propagation delays are the same for different links. Moreover, we assume that $\tau<1$. This is because propagation delay is relatively small compared to the waiting delay.

After introducing propagation delay, $\gamma(\lambda)$ increases obviously. Now, $\gamma(\lambda)>0$ even when $\lambda>\lambda_{I}$. Thus, the second property of $\gamma(\lambda)$ in theorem 3.1 no longer holds. Instead, $\gamma(\lambda)$ is uniformly lower bounded by a constant larger than 0 . And $\gamma(\lambda)$ tends to this uniform lower bound as $\lambda$ goes to infinity. A representation of $\gamma(\lambda)$ is given in Fig. 4.

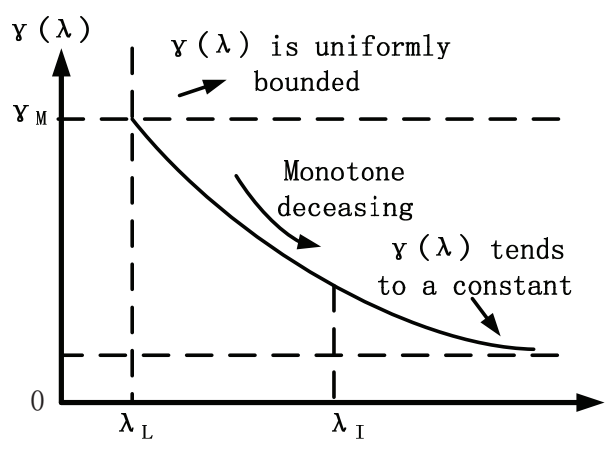

Figure 4: Sketch of $\gamma(\lambda)$ (considering propagation delay).

\footnotetext{
${ }^{6}$ This is because the waiting delay is caused by the lack of instantaneous connectivity of wireless network. When node density is large enough, the wireless network has instantaneous connectivity, making waiting delay negligible.
} 
Now, we present the following theorem. Here, both upper bound and lower bound have taken propagation delay into consideration. The proof of Theorem 4.1 follows similar techniques we used before, thus we omit the proof here.

TheOREM 4.1. Given $R C M \mathcal{G}_{t}\left(\lambda, r_{0}, g(r)\right)$ with $\lambda>\lambda_{L}, \tau$ is the propagation delay for a existing link and $\tau<1$. Then the corresponding $\gamma(\lambda)$ satisfies

$$
\frac{1}{E\left(\min \left\{S_{g}(\lambda), \frac{r_{0}}{\tau}\right\}\right)+r_{0}} \leq \gamma(\lambda) \leq \inf _{\lambda^{\prime} \in\left[\lambda_{L}, \lambda\right]} \frac{\kappa \sqrt{\lambda^{\prime} / \lambda_{L}}}{g\left(r_{0} \sqrt{\frac{\lambda_{L}}{\lambda^{\prime}}}\right)}
$$

where $\kappa$ is a constant independent of $\lambda$.

Proof. We first consider the upper bound. We have already obtained Eqn.(9),i.e.,

$$
\gamma(\lambda) \leq \kappa \sqrt{\frac{\lambda}{\lambda_{L}}} E\left[T_{c}(e)\right]
$$

where the length of link $e$ is smaller than $\tilde{r}=r_{0} \sqrt{\frac{\lambda_{L}}{\lambda}}(1+\epsilon)$.

Using similar method in deriving Eqn. (10), we obtain

$$
\begin{aligned}
E\left[T_{c}(e)\right] & \leq E\left[T_{c}\left(e^{\prime}\right)\right] \\
& =\sum_{k=0}^{\infty}(k+\tau) \mathbb{P}\left(T_{c}\left(e^{\prime}\right)=k\right) \\
& <\sum_{k=0}^{\infty}(k+1)(1-g(\tilde{r}))^{k} g(\tilde{r}) \\
& =\frac{1}{g(\tilde{r})} \\
& =\frac{1}{g\left(r_{0} \sqrt{\frac{\lambda_{L}}{\lambda}}(1+\epsilon)\right)}
\end{aligned}
$$

where $e^{\prime}$ is link whose length is $\tilde{r}$. The inequality above is slightly different from Eqn. (10). This is because we have taken propagation delay into consideration.

Thus,

$$
\gamma(\lambda) \leq \kappa \sqrt{\frac{\lambda}{\lambda_{L}}} \frac{1}{g\left(r_{0} \sqrt{\frac{\lambda_{L}}{\lambda}}(1+\epsilon)\right)} .
$$

Let $\epsilon \rightarrow 0$, we obtain,

$$
\gamma(\lambda) \leq \kappa \sqrt{\frac{\lambda}{\lambda_{L}}} \frac{1}{g\left(r_{0} \sqrt{\frac{\lambda_{L}}{\lambda}}\right)} .
$$

Note that $\gamma(\lambda)$ is a monotone decreasing function, thus

$$
\gamma(\lambda) \leq \inf _{\lambda^{\prime} \in\left[\lambda_{L}, \lambda\right]} \kappa \sqrt{\frac{\lambda^{\prime}}{\lambda_{L}}} \frac{1}{g\left(r_{0} \sqrt{\frac{\lambda_{L}}{\lambda^{\prime}}}\right)} .
$$

Then we consider the lower bound. Similar to the previous part, we still focus on the cluster to cluster transmission. Consider a cluster to cluster transmission process

$$
\Pi=\left\{\left(u^{(1)}, t_{1}\right),\left(u^{(2)}, t_{2}\right), \ldots,\left(u^{(M)}, t_{M}\right)\right\},
$$

similarly, we have, for $\forall k=1,2, \ldots, M-1$,

$$
\left\|u^{(k+1)}-u^{(k)}\right\| \leq S_{g, t_{k}, u^{(k)}}(\lambda)+r_{0} .
$$

And for $k=M$,

$$
\left\|v-u^{(M)}\right\| \leq S_{g, t_{M}, u_{x}^{(M)}}(\lambda)<S_{g, t_{M}, u^{(M)}}(\lambda)+r_{0} .
$$

Besides, the distance transmitted is also limited by the finite hops in one time slot. Since each hop takes $\tau$ time slot, then the message can experience at most $\frac{1}{\tau}$ hops in one time slot. As a result, the longest distance transmitted in one time slot is upper bounded by $\frac{r_{0}}{\tau}$. Then for $\forall k=$ $1,2, \ldots, M-1$,

$$
\left\|u^{(k+1)}-u^{(k)}\right\| \leq \frac{r_{0}}{\tau}+r_{0} .
$$

And for $k=M$,

$$
\left\|v-u^{(M)}\right\| \leq \frac{r_{0}}{\tau}<\frac{r_{0}}{\tau}+r_{0} .
$$

Integrating the above four inequalities, we obtain, for $\forall k=$ $1,2, \ldots, M-1$,

$$
\left\|u^{(k+1)}-u^{(k)}\right\| \leq \min \left\{S_{g, t_{k}, u}(k)(\lambda), \frac{r_{0}}{\tau}\right\}+r_{0} .
$$

And for $k=M$,

$$
\left\|v-u^{(M)}\right\| \leq \min \left\{S_{g, t_{M}, u}(M)(\lambda), \frac{r_{0}}{\tau}\right\}+r_{0} .
$$

Again, using the method in section 4.3, we immediately obtain

$$
\gamma(\lambda) \geq \frac{1}{E\left(\min \left\{S_{g}(\lambda), \frac{r_{0}}{\tau}\right\}\right)+r_{0}} .
$$

Theorem 4.1 formulates the impact of both waiting delay and propagation delay to delay-distance ratio $\gamma(\lambda)$. This makes our results more applicable and more reliable.

\section{SIMULATION}

In this section, we make simulations to uphold our theoretical results. First, we give a further discussion on some parameters in our expressions. Then, enormous simulations are done to justify several assertions in this paper. Our theoretical results are based on a relatively general model, Random Connection Model. Many Network Models can be converted to a Random Connection Model, making our results applicable to many different cases. The difference is that the connection functions are different in different cases. In this paper, we don't specify specific interference model and channel fading model, therefore, we cannot give the exact connection function. As a result, we just randomly choose one for simulation. In the following discussion, we simply let $r_{0}=1$, and the connection function $g$ be defined as

$$
g(r)=\left\{\begin{array}{rll}
\frac{1}{4}(2-r)^{2} & : & r \leq 1 \\
0 & : & r>1
\end{array}\right.
$$

\subsection{Discussion on Several Parameters}

In our expression of theoretical bounds Eqn. (6), two terms $\kappa$ and $E\left(S_{g}(\lambda)\right)$ are applied. Besides, In Eqn. (16), $E\left(\min \left\{S_{g}(\lambda), \frac{r_{0}}{\tau}\right\}\right)$ is applied. It is very hard to get these values mathematically, which might be mathematically intractable. Therefore, we make a compromised to obtain these parameters by simulation. Our goal in this paper is 
to bound a parameter, which takes more effort to calculate(even using simulation), by some other parameters that are easier to obtain(maybe by simulation).

$\kappa$ is a constant, and we simply obtain its value through simulation; while $E\left(S_{g}(\lambda)\right)$ and $E\left(\min \left\{S_{g}(\lambda), \frac{r_{0}}{\tau}\right\}\right)$ are functions depend on $\lambda$, and we find two analytical expressions to approximate them.

We first focus on $\kappa$ (defined in lemma 4.3). In our simulation, we simulated 2304 points in a $40 \times 40$ region. The node density is $\lambda=\lambda_{L} \approx 1.44$, and the transmission range is 1.01. A message is originated from a node located at the center of the region, we record down the minimum number of hops and the distance from the source for each node, and present it in Fig. 5.(a).
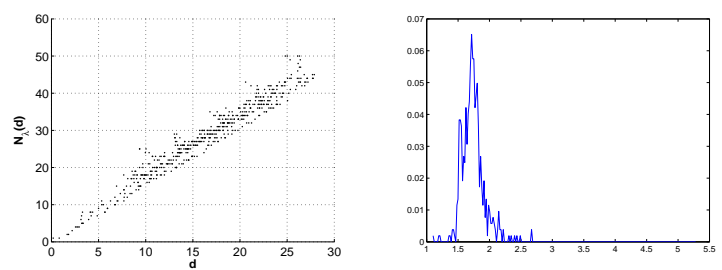

(a) Illustration of the re- (b) The probability distrilationship between $N_{\lambda}(d)$ bution graph of $\frac{N_{\lambda}(d)}{d}$
and $d$

Figure 5: Simulation results on $\kappa$. The first Figure reveals the linear relationship between $N_{\lambda}(d)$ and $d$, and the second indicates $\kappa \approx 1.7153$.

From Fig. 5.(a), we can see that $N_{\lambda}(d)$ grows linearly with $d$. To find $\kappa$, we calculate $\frac{N_{\lambda}(d)}{d}$ for each node, and present its probability distribution graph in Fig. 5.(b). It can be seen from Fig. 5.(b) that the probability $\frac{N_{\lambda}(d)}{d}=1.7153$ is the largest. Thus, $\kappa \approx 1.7153$.

Next, we turn to $E\left(S_{g}(\lambda)\right)$. The physical meaning of $E\left(S_{g}(\lambda)\right)$ is the average size of the connected component intersected with the origin. It is obvious to note that when $\lambda=0, E\left(S_{g}(\lambda)\right)=0$. And when $\lambda=\lambda_{I}, E\left(S_{g}(\lambda)\right)=\infty$ since the network is percolated in this case. Thus, we give a conjecture about the analytical expression of $E\left(S_{g}(\lambda)\right)$, i.e.,

$$
E\left(S_{g}(\lambda)\right)=\frac{c_{1} \lambda}{\lambda_{I}-\lambda} .
$$

We make enormous numerical computations to find the experimental values of $E\left(S_{g}(\lambda)\right)$ with respect to different $\lambda$, ranging from $1.44\left(\lambda_{L} \simeq 1.44\right)$ to 2.4. We then rewrite Eqn. (19) as

$$
\frac{1}{E\left(S_{g}(\lambda)\right)}=\frac{\lambda_{I}}{c_{1}} \cdot \frac{1}{\lambda}-\frac{1}{c_{1}}
$$

Using least square method, we can easily obtain $c_{1} \approx$ $1.2841, \lambda_{I} \approx 2.4886$.

Then we make a comparison between the fitting value and the experimental value of $E\left(S_{g}(\lambda)\right)$. From Fig. 6.(a), It can be seen that there is a good agreement between fitting and experimental results.

Next, we come to estimate $E\left(\min \left\{S_{g}(\lambda), \frac{r_{0}}{\tau}\right\}\right)$. Similar to $E\left(S_{g}(\lambda)\right), E\left(\min \left\{S_{g}(\lambda), \frac{r_{0}}{\tau}\right\}\right)=0$ whenever $\lambda=0$. Moreover, $E\left(\min \left\{S_{g}(\lambda), \frac{r_{0}}{\tau}\right\}\right) \leq \frac{r_{0}}{\tau}$ for $\forall \lambda$, and is monotone increasing with $\lambda$. Thus, we conjecture that the analytical

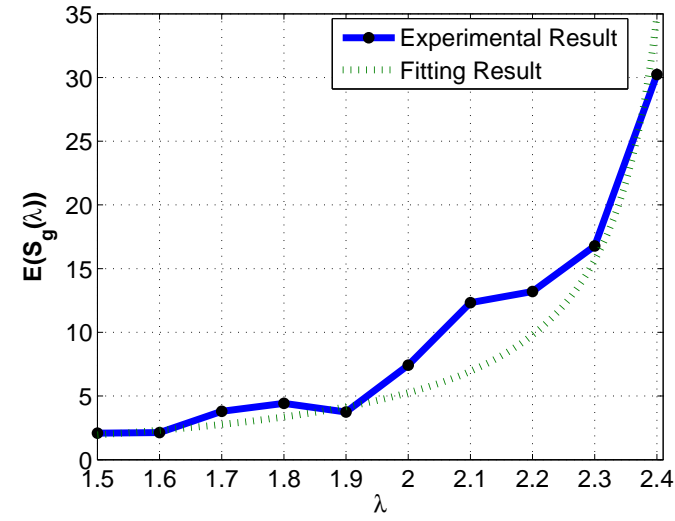

(a) $E\left(S_{g}(\lambda)\right)$

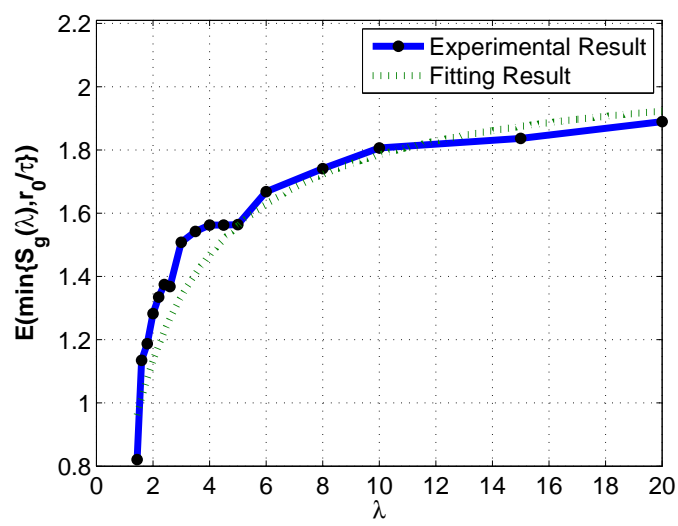

(b) $E\left(\min \left\{S_{g}(\lambda), \frac{r_{0}}{\tau}\right\}\right)$

Figure 6: Comparison between experimental and fitting value of $E\left(S_{g}(\lambda)\right)$ and $E\left(\min \left\{S_{g}(\lambda), \frac{r_{0}}{\tau}\right\}\right)$.

expression of $E\left(\min \left\{S_{g}(\lambda), \frac{r_{0}}{\tau}\right\}\right)$ has the format

$$
E\left(\min \left\{S_{g}(\lambda), \frac{r_{0}}{\tau}\right\}\right)=\frac{c_{2} \lambda}{c_{3}+\lambda} .
$$

Similarly, we make enormous numerical computations to find the experimental values of $E\left(\min \left\{S_{g}(\lambda), \frac{r_{0}}{\tau}\right\}\right)$ with respect to different $\lambda$, ranging from 1.44 to 20 . We rewrite Eqn. (20) as

$$
\frac{1}{E\left(\min \left\{S_{g}(\lambda), \frac{r_{0}}{\tau}\right\}\right)}=\frac{c_{3}}{c_{2}} \cdot \frac{1}{\lambda}+\frac{1}{c_{2}} .
$$

Using least square method, we can easily obtain $c_{2} \approx$ $2.0845, c_{3} \approx 1.6813$.

Then we make a comparison between the fitting value and the experimental value of $E\left(\min \left\{S_{g}(\lambda), \frac{r_{0}}{\tau}\right\}\right)$. From Fig. 6.(b), It can be seen that there is a good agreement between fitting and experimental results.

\subsection{Comparison between Two Bounds}

This paper is originated from the idea that the delaydistance ratio $\gamma(\lambda)$ may depend on the node density $\lambda$. We calculate $\gamma(\lambda)$ under different node densities. From Fig. 7, we can see that $\gamma(1.6)=\lim _{d \rightarrow \infty} \frac{T_{\lambda}(d)}{d} \approx 0.68, \gamma(1.9) \approx$ 
$0.35, \gamma(2.2) \approx 0.11, \gamma(2.5) \approx 0$. This justify the fact that $\gamma(\lambda)$ depend on $\lambda^{7}$, making our discussion meaningful.

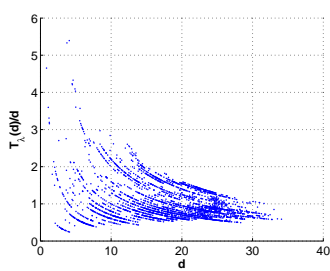

(a) $\lambda=1.6$

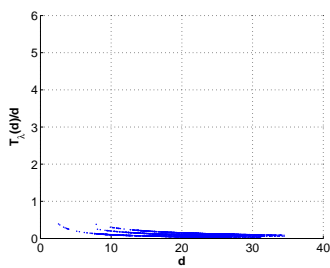

(c) $\lambda=2.2$

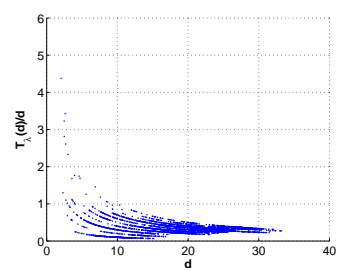

(b) $\lambda=1.9$

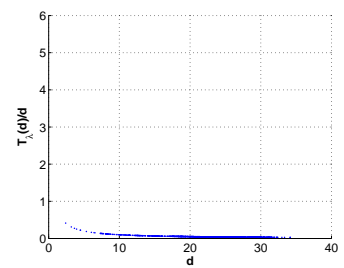

(d) $\lambda=2.5$
Figure 7: Simulation results on different $\lambda$.

Now, we are ready to compare our theoretical bounds and the experimental values of $\gamma(\lambda)$. We first ignore the propagation delay, Fig. 8 shows the comparison between the experimental value and our theoretical value of both the upper bound and the lower bound. In our simulations, We work out the experimental values of $\gamma(\lambda)$ where $\lambda$ is set to be evenly distributed in the interval of $\left[\lambda_{L}, \lambda_{I}\right]$. From Fig. 8, we find the experimental values are right bounded by both the upper and the lower bounds.

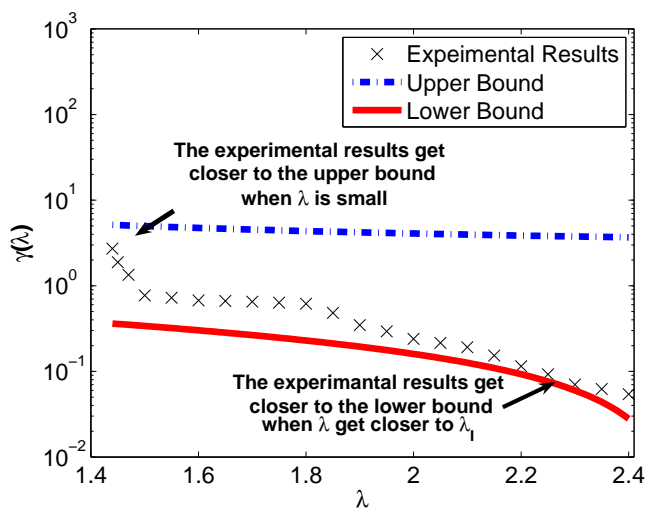

Figure 8: Comparison between upper bound and lower bound(propagation delay is ignored).

Then, we take propagation delay into consideration. In the following part, we take $\tau=0.2$. We first examine the effect of introducing propagation delay to the delay-distance ratio $\gamma(\lambda)$.

We consider two networks, whose node densities $\lambda$ are 1.5 and 2.2(1.5 is near $\lambda_{L}, 2.2$ is near $\left.\lambda_{I}\right)$ respectively. For each

\footnotetext{
${ }^{7}$ To be more exact, $\gamma(\lambda)$ decreases with $\lambda$.
}

network, we make simulations to find $\gamma(\lambda)$ in the cases $\tau=0$ and $\tau=0.2$. The result is show in Fig. 9 .
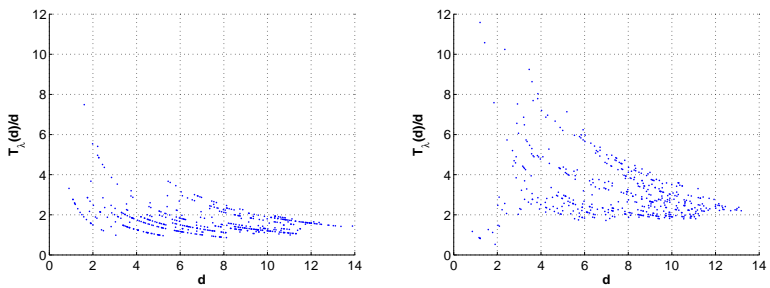

(a) $\lambda=1.5, \tau=0, \gamma(\lambda) \approx(\mathrm{b}) \lambda=1.5, \tau=0.2, \gamma(\lambda) \approx$ 1.8

2.1
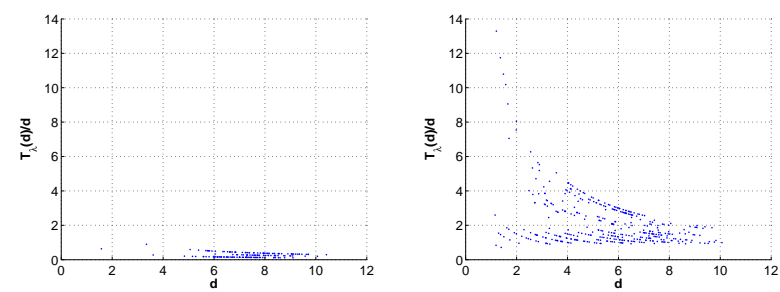

(c) $\lambda=2.2, \tau=0, \gamma(\lambda) \approx(\mathrm{d}) \lambda=2.2, \tau=0.2, \gamma(\lambda) \approx$ 0.1

1.8

Figure 9: The influence of propagation delay $\tau$ to the delay-distance ratio $\gamma(\lambda)$.

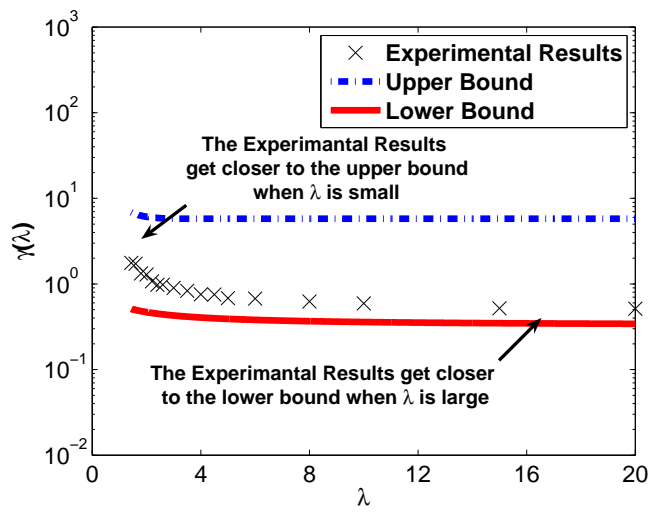

Figure 10: Comparison between upper bound and lower bound(propagation delay is considered).

From Fig. 9, we can also see that when $\lambda$ is small, the influence of $\tau$ to $\gamma(\lambda)$ is small; and the influence becomes more significant as $\lambda$ grows larger. This also indicate that when node density is small, delay is mainly caused by the waiting delay(waiting delay is mainly caused by the lack of instantaneous connectivity); while when node density is large, delay is mainly caused by propagation delay.

Next, we compare our theoretical bounds and experimental results of $\gamma(\lambda)$ in the case $\tau=0.2$. In our simulation, the node densities $\lambda$ are chosen from $[1.44,20]$. As the change of $\gamma(\lambda)$ is larger when $\lambda$ is small, we choose more simulation points for smaller node density. The comparison between our theoretical bounds and experimental results is shown in 
Fig. 10. The experimental values are right bounded by both the upper and the lower bounds.

From Fig. 8 and Fig. 10, it can be also seen that when $\lambda$ is large, $\gamma(\lambda)$ is much closer to the lower bound. An explanation to this phenomena is that the larger $\gamma(\lambda)$ is, the larger the size of clusters in the cluster to cluster transmission process. Larger cluster size provides more opportunity to forward messages. Thus, when the node density is large enough, it is probably that the message can transmit again right at the next time slot. This makes our lower bound more accurate. However, our upper bound is a little bit loose. But we can see that $\gamma(\lambda)$ gets closer to the upper bound when the node density $\lambda$ decreases. This is because our upper bound is obtained from the delay of just one path. And the smaller $\lambda$ is, the smaller the number of paths connecting two nodes. This makes $\gamma(\lambda)$ get closer to the upper bound.

\section{CONCLUSION}

In this paper, we study the tradeoff between $\gamma(\lambda)$ and $\lambda$ using percolation theory. We point out that the lack of instantaneous connectivity incurs waiting delay and prove that $\gamma(\lambda)$ is upper bounded by $\inf _{\lambda^{\prime} \in\left[\lambda_{L}, \lambda\right]} \kappa \sqrt{\frac{\lambda^{\prime}}{\lambda_{L}}}\left(\frac{1}{g\left(r_{0} \sqrt{\frac{\lambda_{L}}{\lambda^{\prime}}}\right)}-1\right)$, and lower bounded by $\frac{1}{E\left(S_{g}(\lambda)+r_{0}\right)}$. Then we take propagation delay into consideration, and obtain further results. Finally, through simulations based on the exact value of $\gamma(\lambda)$, we further obtain a new observation that the lower bound serves as a good estimate to the value of $\gamma(\lambda)$ in dense networks. And $\gamma(\lambda)$ gets closer to the upper bound when $\lambda$ decreases. Simulation results conform our theoretical findings.

\section{Acknowledgements}

This work is supported by National Basic research grant (2011CB302701), NSF China (No. 60832005); China Ministry of Education Fok Ying Tung Fund (No.122002); Qualcomm Research Grant; National Key Project of China (2009ZX03003-006-03, 2010ZX03003-001-01); National High tech grant of China (2009AA01Z248).

\section{REFERENCES}

[1] J.M.Hammersley and D.J.A.Welsh, "First Passage Percolatin, Subadditive Processes, Stacastic Networks, and Generalized Renewal Theory," in Proc. Internat. Res. Semin., Statist. Lab., Univ. California, Berkeley, Calif., pages 61-110. Springer-Verlag, New York, 1965.

[2] Z. Kong and E. M. Yeh, "On the Latency for Information Dissemination in Mobile Wireless Networks," in Proc. ACM MobiHoc'08, Hong Kong SAR, China, May. 2008.

[3] P. Gupta and P. R. Kumar, "Critical Power for Asymptotic Connectivity in Wireless Networks," in Stochastic Analysis, Control, Optimization and Applications: A Volume in Honor of W.H. Fleming, W. M. McEneany, G. Yin, Q. Zhang, Eds. Boston, MA: Birkhauser, pp. 547-566, 1998.

[4] P. Wan and C. W. Yi, "Asymptotic Critical Transmission Radius and Critical Neighbor Number for K-connectivity in Wireless Ad Hoc Networks," in Proc. 5th ACM int. symp. Mobile Ad Hoc Networking and Computing, Roppongi Hills, Tokyo, Japan, May 24-26, 2004.

[5] M. D. Penrose, "On K-connectivity for a Geometric Random Graph," Random Structure and Algorithms, vol. 15, no. 2, pp. 145-164, Sept. 1999.

[6] Q. Wang, X. Wang and X. Lin, "Mobility Increases the Connectivity of K-hop Clustered Wireless Networks," ACM Mobicom'09, Beijing, Sept. 2009.

[7] D. Goeckel, B. Liu, D. Towsley, L. Wang and C. Westphal, "Asymptotic connectivity properties of cooperative wireless ad hoc network," $A C M$ Mobihoc'08, May. 2008.

[8] O. Dousse, F. Baccelli and P. Thiran, "Impact of Interferences on Connectivity in Ad Hoc Networks," in Proc. of IEEE Infocom, pp. 1724-1733, Apr. 2005.

[9] G. R. Grimmett, "Percolaiton," Springer, 1999.

[10] O. Dousse, P. Mannersalo and P. Thiran, "Latency of Wireless Sensor Networks with Uncoordinated Power Saving Mechnisms," in Proc. ACM MobiHoc'04, pp. 129-120, May. 2004.

[11] Z. Kong, and E. M. Yeh. "Connectivity and Information Dissemination in Large-Scale Wireless Networks with Dynamic Links," submitted to IEEE Trans. Inform. Theory, 2008.

[12] D. J. Daley and D. Vere-Jones, "An Introduction to the Theory of Point Process," New York: Springer, 1988.

[13] J. F. C. Kingman, "Poisson Processes," Clarendon Press, Oxford, 1993.

[14] R. Meester and R. Roy, "Continuum Percolation," NewYork: Cambridge University Press, 1996.

[15] T. Liggett, "An Improved Subadditive Ergodic Theorem," Annals of Prob., vol. 13, pp. 1279-1285, 1985.

[16] W. Ren, Q. Zhao, and A. Swami, "On the Connectivity and Multihop Delay of Ad Hoc Cognitive Radio Networks" to appear in Proc. of IEEE International Conference on Communications(ICC), May, 2010.

[17] S. Zhao, L. Fu, X. Wang, Q. Zhang, "Tradeoff between Node Density and Delay in Wireless Networks with Unreliable Links" Technical Report, 2010. Available at http://iwct.sjtu.edu.cn/Personal/xwang8/paper/MO BICOM2011_Tech.pdf. 